\title{
TRCI50094 attenuates progression of nontraditional cardiovascular risk factors associated with obesity and type 2 diabetes in obese ZSFI rats
}

This article was published in the following Dove Press journal: Diabetes, Metabolic Syndrome and Obesity:Targets and Therapy 31 December 2010

Number of times this article has been viewed

\author{
Shitalkumar P Zambad' \\ Siralee Munshi² \\ Amita Dubey ${ }^{3}$ \\ Ram Gupta' \\ Rosa Anna Busiello 4 \\ Antonia Lanni ${ }^{5}$ \\ Fernando Goglia ${ }^{6}$ \\ Ramesh C Gupta ${ }^{7}$ \\ Vijay Chauthaiwale ${ }^{8}$ \\ Chaitanya Dutt ${ }^{9}$
}

'Pharmacology, ${ }^{2} \mathrm{Cellular}$ and Molecular Biology, ${ }^{3}$ Pre-clinical and Safety Evaluation, Torrent Research Centre, Torrent Pharmaceuticals Ltd, Gujarat, India; ${ }^{4}$ Dipartimento di Biologia, Universita degli Studi di Napoli Federico II, Naples, Italy; ${ }^{5}$ Dipartimento di Scienze della Vita, Seconda Universita di Napoli, Caserta, Italy; ${ }^{6}$ Dipartimento di Scienze Biologiche ed Ambientali, Universita del Sannio, Benevento, Italy; ${ }^{7}$ Medicinal Chemistry, ${ }^{8}$ Discovery Research, ${ }^{9} \mathrm{Clinical}$ Research, Torrent Research Centre, Torrent Pharmaceuticals Ltd, Gujarat, India

Correspondence: Chaitanya Dutt Torrent Research Centre, PO Bhat, Dist Gandhinagar 382428, Gujarat, India

Tel +91 7923969100

Fax +91 7923969135

Email cdutt@torrentpharma.com

\begin{abstract}
Chronic overnutrition and consequential visceral obesity is associated with a cluster of risk factors for cardiovascular disease and type 2 diabetes mellitus. Moreover, individuals who have a triad of hypertension, dysglycemia, and elevated triglycerides along with reduced highdensity lipoprotein cholesterol have a greater residual cardiovascular risk even after factoring for the traditional risk factors such as age, smoking, diabetes, and elevated low-density lipoprotein cholesterol. In our previous study we demonstrated that TRC150094, when administered to rats receiving a high-fat diet, stimulated mitochondrial fatty acid oxidation (FAO) and reduced visceral adiposity, opening an interesting perspective for a possible clinical application. In the present study, oral administration of TRC150094 to obese Zucker spontaneously hypertensive fatty rats (obese ZSF1) improved glucose tolerance and glycemic profile as well as attenuated a rise in blood pressure. Obese ZSF1 rats treated with TRC150094 also showed reduced hepatic steatosis, reduced progression of nephropathy, and improved skeletal muscle function. At the cellular level, TRC150094 induced a significant increase in mitochondrial respiration as well as an increased FAO in liver and skeletal muscle, ultimately resulting in reduced hepatic as well as total body fat accumulation, as evaluated by magnetic resonance spectroscopy and magnetic resonance imaging, respectively. If reproduced in humans, these results could confirm that TRC150094 may represent an attractive therapeutic agent to counteract multiple residual cardiovascular risk components.
\end{abstract}

Keywords: CV risk factors, energy expenditure, fatty acid oxidation, obesity, TRC150094, type 2 diabetes

\section{Introduction}

Excess caloric intake and/or a sedentary lifestyle are the obvious culprits that lead to obesity and insulin resistance, which eventually progresses to type 2 diabetes mellitus. ${ }^{1}$ Not all obese individuals carry an increased cardiovascular (CV) risk beyond the traditional risk factors; however, patients in whom a cluster of several nontraditional risk factors associated with $\mathrm{CV}$ disease occur together, carry a relatively higher absolute risk of CV events. ${ }^{2}$ This cluster includes ectopic lipid deposition exemplified by nonalcoholic fatty liver disease, ${ }^{3-5}$ carbohydrate intolerance, ${ }^{6}$ atherogenic dyslipidemia, ${ }^{7}$ and hypertension. ${ }^{8}$ Several studies have reported that individuals who have a triad of hypertension, dysglycemia, and elevated triglycerides along with reduced high-density lipoprotein (HDL) cholesterol (atherogenic dyslipidemia) have a greater residual CV risk even after factoring for the traditional risk factors such as age, smoking, diabetes, 
and elevated low-density lipoprotein (LDL) cholesterol.9-11 Regular physical activity and reduction of caloric intake represent the ideal strategy to counteract this syndrome, ${ }^{12}$ which is rarely achieved in real life. Therefore, much effort is being devoted to develop agents useful in counteracting the aforementioned metabolic abnormalities. Current standards of care for $\mathrm{CV}$ disease prevention emphasize the importance of multifactorial intervention to achieve recommended targets for LDL cholesterol, blood pressure, and glycemic control. ${ }^{13,14}$ Although several treatment options exist for individual components of this cluster, an optimal approach aimed at correcting the metabolic derangement is of paramount importance in order to target residual CV risks. ${ }^{15}$

TRC150094 is a novel synthetic compound that displays the capacity to stimulate energy expenditure. In our recently published study, we have demonstrated that TRC150094 increases whole body energy expenditure, increases mitochondrial fatty acid oxidation (FAO), and reduces abdominal adiposity in rats fed a high-fat diet. ${ }^{16}$ These findings provoked the evaluation of the potential of TRC150094 for the treatment of a cluster of multiple CV risk factors associated with visceral obesity and associated metabolic derangement. Hence, the present study was designed to evaluate the effects of TRC150094 in an animal model that spontaneously displays a phenotype akin to patients with the aforementioned cluster of nontraditional $\mathrm{CV}$ risk factors.

In the present study, we have employed obese ZSF1 rats, an animal model that spontaneously develops obesity and dysglycemia as well as an associated clinically relevant constellation of comorbidities. Indeed, adult obese ZSF1 rats exhibit many of the features common to human metabolic syndrome, including obesity, hyperglycemia, insulin resistance, hypertension, and severe dyslipidemia. ${ }^{17}$

\section{Material and methods}

\section{Animals, grouping, and treatment}

Male obese ZSF1 rats (Charles River Laboratories, Wilmington, MA, USA) 12 weeks in age $(n=30)$ were used for the study. Principles of laboratory animal care, ${ }^{18}$ as well as Committee for the Purpose of Control and Supervision of Experiments on Animals guidelines for animal care ${ }^{19}$ were followed. Rats were housed in individually ventilated cage systems, maintained on a 12-hour light-dark cycle, and had access to Purina 5008 rat chow and water ad libitum. The individually ventilated cages were maintained under a controlled environment of temperature $22 \pm 3{ }^{\circ} \mathrm{C}$, relative humidity $30 \%-70 \%$, and air exchange rate $40-50$ air changes per hour. Some of the rats $(n=10)$ were implanted with radiotelemetry implants (TL11M2C50PXT, Data Sciences, St Paul, MN, USA) at the age of 6-8 weeks. At the age of 3 months, all obese ZSF1 rats were divided into three groups: control, $6 \mathrm{mg} / \mathrm{kg}$ (receiving TRC150094 $6 \mathrm{mg} / \mathrm{kg} / \mathrm{d}$ orally twice daily), and $12 \mathrm{mg} / \mathrm{kg}$ (receiving TRC150094, $12 \mathrm{mg} / \mathrm{kg} / \mathrm{d}$ orally twice daily). The grouping was done such that the control and $12 \mathrm{mg} / \mathrm{kg}$ groups included five rats per group that were implanted with radiotelemetry implants.

\section{Blood pressure recording by radiotelemetry}

The influence of long-term treatment on blood pressure, one of the very relevant triad components associated with $\mathrm{CV}$ risk in metabolic syndrome, was measured using a radiotelemetry system (Dataquest A.R.T. 3.01, Data Sciences International, St Paul, MN, USA). Scheduled rat cages were placed on top of a receiver (RPC1, Data Sciences) for measurement of blood pressure, which was recorded from rats implanted with TL11M2C50PXT transmitters. Data were collected with a computer-driven data acquisition system (Dataquest A.R.T. 3.01, Data Sciences); the acquisition software was appropriately configured to record parameters for 30 seconds every 10 minutes, continuously for 24 hours. The systolic blood pressure (SBP) and diastolic blood pressure (DBP) data acquired were analyzed and represented as light time (mean of daytime data) and dark time (mean of nighttime data) blood pressure.

\section{Body fat and liver fat measurement}

A magnetic resonance imaging (MRI) technique was used to assess the influence of TRC150094 on whole body fat and liver fat accumulation. For MRI and magnetic resonance spectroscopy (MRS) acquisition, anesthesia was induced by inhalation of a mixture of oxygen and 5\% isoflurane and maintained with a mixture of oxygen containing $2 \%-2.5 \%$ isoflurane. Anesthetized obese ZSF1 rats were placed in a supine position in a quadrature transmit/receive head coil (length $28 \mathrm{~cm}$ and height $26 \mathrm{~cm}$ ). All MRI/MRS experiments were carried out using a Philips model, ACHIEVA, equipped with a 3 Tesla horizontal magnet with a $60 \mathrm{~cm}$ bore. View forum R6-1V1L2SP 2007 was used to analyze data.

For measurement of total body fat ( $n=6$ rats per group), radiofrequency pulses were transmitted, and the nuclear magnetic resonance signal was acquired with quadrature transmit/receive head coil. Contiguous transversal slices were obtained along the rat length using a T1 weighted-spin echo and fat suppress technique with 128 phase-encoding steps. The imaging sequence was optimized for short echo 
time (echo time 15 milliseconds) and short repetition time (repetition time 673 milliseconds) to allow for signal suppression from tissues other than fat. Usually, 3-mm-thick coronal slices were obtained of a total of 20 images to cover the whole body with FOV $259 \mathrm{~mm}$ and NEX-2. Two-dimensional image series were then imported to view forum R6-1V1L2SP 2007 Philips software for estimation of fat volumes.

A stimulated echo acquisition method (SV-STEAM) was used in conjunction with CHESS sequence to suppress the water signals for MRS acquisition. The acquisition parameters were: 2000/21 (repetition time milliseconds/echo time milliseconds), $2000 \mathrm{~Hz}$ sweep width, 1024 data points, and 128 images. Total acquisition time with respiratory trigger was 25 minutes.

For measurement of hepatic fat by MRS in obese ZSF1 rat SV-STEAM spectra were acquired in the supine position. A localization voxel of $5 \mathrm{~mm}^{3}$ was placed in the right hepatic lobe of the liver, adjacent to the portal vein near the edge of the liver, with care taken to avoid the large intrahepatic vessels. To diminish tissue contamination from the adjacent structures and to maintain proper localization, all voxels were positioned by individuals experienced in abdominal radiology. A respiratory trigger was applied longitudinally over the body surface to avoid artifacts due to irregular respiration.

\section{Oral glucose tolerance and fasting plasma biochemical parameters}

An oral glucose tolerance test (OGTT) was performed at week 6 and week 24 . At time 0 minutes, blood collection was performed in 8-hour-fasted obese ZSF1 rats under light isoflurane anesthesia from the sublingual vein. Glucose was then administered orally at a dose of $2 \mathrm{~g} / \mathrm{kg}$ with a dose volume of $4 \mathrm{~mL} / \mathrm{kg}$. Blood was collected at 15, 30, 60, and 120 minutes after glucose load. Plasma was separated for estimation of glucose. Blood samples were collected from 8-hour-fasted obese ZSF1 rats for measurement of fasting plasma glucose, cholesterol, and triglycerides at weeks 0, 6, 16, and 24 . Plasma fructosamine levels were measured from the blood samples collected at treatment week 24 . For measurement of random plasma glucose levels, blood samples were collected at 8.00 a.m. from nonfasted rats at weeks 0,5 , and 24 .

\section{Effect on forelimb grip strength}

The forelimb grip strength in rats was measured using a Grip Strength Meter (Columbus Instruments International, Columbus, OH, USA). Rats were held by the scruff of the neck with one hand, and at the base of the tail with the other. The forepaws were located within the pull bar connected to the force gauges and the rat slowly pulled away from the pull bar until it released the pull bar.

\section{Measurement of mitochondrial respiratory parameters, FAO, and carnitine palmitoyl transferase activity assay}

In our previous study, in Wistar rats fed a high-fat diet it was demonstrated that TRC150094 influences the mitochondrial substrate oxidation in skeletal muscle and liver. ${ }^{16}$ In our present study, we also monitored terminally the mitochondrial respiratory parameters, FAO, and carnitine palmitoyl transferase (CPT) activity assay to assess whether chronic administration of TRC150094 likewise influences the mitochondrial substrate oxidation capacity in obese ZSF1 rats that maintained on a relatively high carbohydrate diet.

\section{Isolation of mitochondria}

At the end of treatment duration, rats were euthanized and tissues such as liver and hind limb skeletal muscles were excised and minced in an ice-cold buffer consisting of $220 \mathrm{mM}$ mannitol, $70 \mathrm{mM}$ sucrose, $20 \mathrm{mM}$ Tris- $\mathrm{HCl}, 1 \mathrm{mM}$ ethylenediaminetetraacetic acid (EDTA), and $5 \mathrm{mM}$ ethylene glycol tetraacetic acid (EGTA) ( $\mathrm{pH} 7.4$ ), and then homogenized in a Potter-Elvehjem homogenizer. Nuclei and cell debris were removed by centrifugation at $500 \mathrm{~g}$ for 10 minutes, with the resulting supernatant being centrifuged at $3000 \mathrm{~g}$ for liver and $8000 \mathrm{~g}$ for skeletal muscle. ${ }^{21}$ The mitochondrial pellet was washed twice and resuspended in a minimal volume of isolation medium and kept on ice until used. The protein concentration was determined by the Hartree method, using bovine serum albumin (BSA) as standard.

\section{Mitochondrial respiration}

Succinate-induced state 4 respiration (uncoupled respiration in which there is no phosphorylation of adenosine diphosphate [ADP] to adenosine triphosphate) and state 3 respiration (coupled respiration in which phosphorylation of ADP is at the maximal rate) of isolated mitochondria $(0.2 \mathrm{mg})$ were determined polarographically at $37^{\circ} \mathrm{C}$ using a Clark-type electrode (Oxytherm, Hansatech Instruments Limited, Norfolk, UK) in $1 \mathrm{~mL}$ of respiratory medium consisting of $80 \mathrm{mM} \mathrm{KCl}, 50 \mathrm{mM}$ 4-(2-hydroxyethyl)-1piperazineethanesulfonic acid (HEPES), $1 \mathrm{mMEGTA,} 1 \mathrm{mM}$ EDTA, $5 \mathrm{mM} \mathrm{KH}_{2} \mathrm{PO}_{4}, 2 \mathrm{mM} \mathrm{MgCl}, 5 \mathrm{mM}$ succinate, $4 \mu \mathrm{M}$ rotenone, and 1\% BSA ( $\mathrm{pH} 7.0)$. Rotenone was added to inhibit complex I of the electron transport chain. 


\section{Measurement of $\mathrm{FAO}$ rate}

The rate of mitochondrial $(0.5 \mathrm{mg})$ FAO was assessed polarographically using a Clark-type electrode at $30^{\circ} \mathrm{C}$ in a final volume of $1 \mathrm{~mL}$ of $80 \mathrm{mM} \mathrm{KCl}, 50 \mathrm{mM}$ HEPES (pH 7.0), 1 mM EGTA, $5 \mathrm{mM} \mathrm{K} \mathrm{HPO}_{4}, 1 \% \mathrm{BSA}(\mathrm{w} / \mathrm{v})$, and $2.5 \mathrm{mM}$ malate in the presence of ADP $(200 \mu \mathrm{M})$. The reaction was started by the addition of palmitoyl coenzyme $\mathrm{A}(\mathrm{CoA})$ in the presence of $2 \mathrm{mM}$ carnitine, the oxidation of which requires its import into the mitochondrion, an influx mediated by CPT or with palmitoyl-carnitine CPTindependent FAO.

\section{CPT activity assay}

To measure $\mathrm{CPT}$ activity, frozen $\left(-70^{\circ} \mathrm{C}\right)$ mitochondria were used. Total CPT (CPT1 plus CPT2) activity was measured spectrophotometrically $(412 \mathrm{~nm})$ by following the kinetics of $2 \mathrm{mM}$ carnitine-dependent CoASH production in the

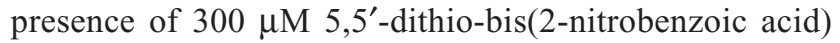
using $50 \mu \mathrm{M}$ palmitoyl-CoA as a substrate. The reaction of palmitoyl CoA in the presence of beta dystrobrevin (DTNB) indicates the involvement of other mitochondrial enzymes.

\section{Citrate synthase activity measurement}

Citrate synthase activity was measured from freshly isolated mitochondria from skeletal muscle by an assay kit from Sigma (CS0720). Assay was performed using acetyl CoA, oxaloacetate, and DTNB. Production of CoASH was measured spectrophotometrically at $412 \mathrm{~nm}$.

\section{Histopathological evaluation}

Sections of liver and kidney tissues were fixed in 10\% neutral buffer formalin for histopathology evaluation. After 3-4 days of fixation, $2-3 \mathrm{~mm}$ thick tissues were trimmed and processed for paraffin sectioning. Four-micron-thick 2-3serial sections were taken from Leica microtome. Transverse sections of kidney were stained with routine hematoxylin and eosin, Periodic acid Schiff (PAS), and Masson's trichrome stain. ${ }^{22}$ The PAS-stained kidney sections were evaluated for nephropathic, tubulopathic, and glomerulosclerotic changes on a scale of grade 0 to $4 .{ }^{17,20,23}$

Liver was microscopically examined in hematoxylin and eosin and PAS-stained sections ${ }^{22}$ on the grade scale of 0 to 4 . In addition to this, a formalin-fixed cryosection of liver was also stained with Churukian Oil Red method ${ }^{20}$ for confirmation of hepatosteatosis. All histopathological evaluation was performed on coded slides.

All studies consisted of $\mathrm{n} \geq 4$ rats per group. Statistics were conducted using Student's $t$-test $\left({ }^{*} P<0.05\right.$, $* * P<0.01, * * * P<0.1$ vs control) and analysis of variance (ANOVA) followed by Dunnett's test $\left({ }^{\sharp} P<0.05,{ }^{\#} P<0.1\right.$ vs control). Statistical significance was defined as one-sided $P$ values.

\section{Results \\ Effect on visceral fat accumulation}

Whole body fat was measured by MRI, and liver fat was quantified by MRS. TRC150094 treatment significantly attenuated liver fat accumulation, which represents a reduction of visceral fat accumulation (Table 1).

The control group showed increased body fat accumulation with age progression, whereas TRC150094-treated male obese ZSF1 rats showed a significant lower fat accumulation (Table 1), which was evident from week 4 of treatment.

\section{Effect on steatohepatitis}

Male obese ZSF1 rats developed steatohepatitis as indicated by a progressive rise in liver fat accumulation and plasma bilirubin with age. TRC150094 treatment resulted in lower levels of plasma bilirubin (at 24 weeks: 2 -fold increase in the TRC150094 $12 \mathrm{mg} / \mathrm{kg}$-treated group vs 8-fold increase in the control group with respect to pretreatment levels) along with

Table I Effect of TRCI50094 on body fat and liver fat in male obese ZSFI rats

\begin{tabular}{|c|c|c|c|c|}
\hline Parameter & $\begin{array}{l}\text { Treatment } \\
\text { week }\end{array}$ & Control & $\begin{array}{l}\text { TRCI } 50094 \\
6 \mathrm{mg} / \mathrm{kg}\end{array}$ & $\begin{array}{l}\text { TRCI } 50094 \\
12 \mathrm{mg} / \mathrm{kg}\end{array}$ \\
\hline \multirow[t]{4}{*}{ Body fat $\left(\mathrm{mm}^{3}\right)$} & 0 & $220 \pm 19.6$ & $237 \pm 25.6$ & $229 \pm 14.5$ \\
\hline & 4 & $330 \pm 30.3$ & $27 I \pm 24.1 * *$ & $270 \pm 17.8^{* *,+}$ \\
\hline & 16 & $290 \pm 29.6$ & $257 \pm 12.2$ & $253 \pm 30.1$ \\
\hline & 24 & $287 \pm 11.2$ & $244 \pm 13 . I^{* \#}$ & $268 \pm 17.2$ \\
\hline \multirow[t]{4}{*}{ Liver fat (peak area, ppm) } & 0 & $199 \pm 11.8$ & $173 \pm 15.0$ & $190 \pm 27.2$ \\
\hline & 4 & $259 \pm 22.4$ & $188 \pm 27.9 * *$ & $186 \pm 28.8 * * \#$ \\
\hline & 16 & $304 \pm 44.6$ & $174 \pm 15 . I^{* \#}$ & $160 \pm 46.3 * \#$ \\
\hline & 24 & $315 \pm 35.1$ & $176 \pm 35.2$ & $174 \pm 59.6$ \\
\hline
\end{tabular}

Notes: Values are mean \pm SEM. ${ }^{*} P<0.05$; ${ }^{*} \mathrm{P}<0.1$ vs control group (Student's $t$-test); ${ }^{*} \mathrm{P}<0.1$ vs control (ANOVA followed by Dunnett's post hoc test), $\mathrm{n} \geq 5$. Abbreviations: ANOVA, analysis of variance; ppm, parts per million; SEM, standard error of mean. 


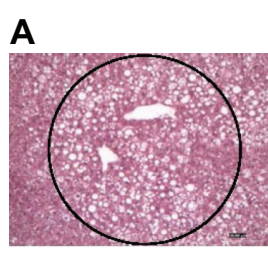

D

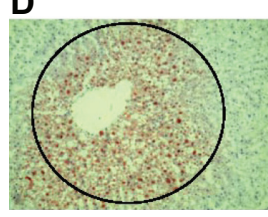

B

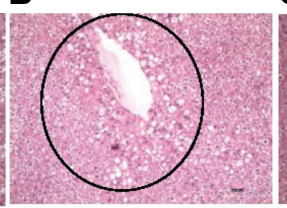

$\mathbf{E}$

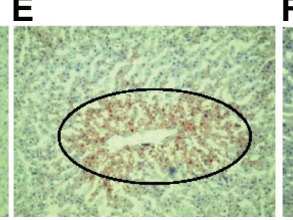

C

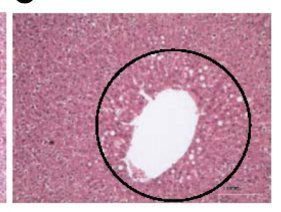

$\mathbf{F}$

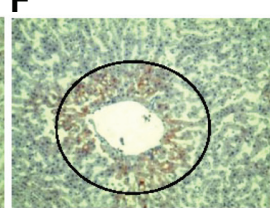

Figure I A) Hematoxylin and eosin D) Oil Red O liver sections of a controltreated obese ZSFI rat showing a high number of hepatocytes with fat droplets; B) Hematoxylin and eosin E) Oil Red O liver sections of a TRCI50094 (6 mg/kg body weight)-treated obese ZSFI rat showing fewer hepatocytes with fat droplets; C) Hematoxylin and eosin F) Oil Red O liver sections of a TRCI50094 (I2 mg/kg body weight)-treated obese ZSFI rat showing the least number of hepatocytes with fat droplets (100× magnification).

a concurrent reduction in liver fat accumulation as observed in MRS and terminal histopathological evaluation (Figure 1), indicating attenuation of steatohepatitis.

\section{Effect on glycemic profile}

Visceral fat accumulation is associated with insulin resistance and diabetes. Hence, effects of TRC150094 on various glycemic parameters were evaluated in obese ZSF1 rats. At the age of 7 weeks, male obese ZSF1 rats were hyperglycemic and hyperinsulinemic. TRC150094 treatment resulted in a significant reduction in fasting hyperglycemia, reduced $\mathrm{AUC}_{\text {glucose }}$ (area under the glucose response curve) during OGTT, and improved plasma fructosamine levels (Table 2). This demonstrates that TRC150094 treatment enhanced glucose utilization and improved glycemic profile.

\section{Effect on blood pressure}

In obese ZSF1 rats, blood pressure rises as age progresses. TRC150094 treatment attenuated a rise in blood pressure with progression of age. In the TRC150094-treated group, progression of both SBP and DBP were significantly lower as compared with the control group $(P<0.01$, repeated-measures ANOVA, Figure 2A and 2B).

\section{Effect on nephropathy}

Male obese ZSF1 rats exhibited symptoms of progressive diabetic nephropathy from the age of 12 weeks. Histopathological observations of the kidney tissue indicated that TRC150094 $12 \mathrm{mg} / \mathrm{kg}$ treatment in obese ZSF1 rats reduced progression of nephropathy and glomerulosclerosis (Figure 3A and 3B).

At 6 and 16 weeks of treatment, TRC150094 $12 \mathrm{mg} / \mathrm{kg}$ reduced the extent of glycosuria by 285 and $1832 \mathrm{mg} /$ day and proteinuria by 12 and $120 \mathrm{mg} /$ day, respectively. Urinary microalbumin was also less by 285 and $1832 \mathrm{mg} /$ day in the TRC150094 $12 \mathrm{mg} / \mathrm{kg}$ group than in the control group at 6 and 16 weeks of treatment, respectively. These findings further substantiated the aforementioned histopathological observations of slow progression of nephropathy in the TRC150094-treated group.

\section{Effect on skeletal muscle function}

About a 117\% increase in citrate synthase activity, a marker of aerobic capacity, and mitochondrial density in skeletal muscle, was observed in the gastrocnemius muscle of male obese ZSF1 rats in the TRC150094-treated group $2.31 \pm 0.57 \mu \mathrm{mol} /$ $\mathrm{mg} /$ minute vs $1.1 \pm 0.3 \mu \mathrm{mol} / \mathrm{mg} /$ minute in the control group. Also, muscle grip strength, a functional parameter of skeletal muscle, was found to be better preserved in the

Table 2 Effect of TRCI50094 on glycemic parameters in male obese ZSFI rats

\begin{tabular}{|c|c|c|c|c|}
\hline Parameter & $\begin{array}{l}\text { Treatment } \\
\text { week }\end{array}$ & Control & $\begin{array}{l}\text { TRCI } 50094 \\
6 \mathrm{mg} / \mathrm{kg}\end{array}$ & $\begin{array}{l}\text { TRCI } 50094 \\
\text { I } 2 \mathrm{mg} / \mathrm{kg}\end{array}$ \\
\hline Fasting plasma & 0 & $286.3 \pm 16.7$ & $292.7 \pm 17.4$ & $280.6 \pm 15.8$ \\
\hline \multirow[t]{3}{*}{ glucose $(\mathrm{mg} / \mathrm{dL})$} & 6 & $328.2 \pm 20.4$ & $299.3 \pm 24.3^{*}$ & $250.4 \pm 21.9 *, \#$ \\
\hline & 16 & $294.6 \pm 15.1$ & $243.6 \pm 14.5^{*} \#$ & $269.2 \pm 10.9$ \\
\hline & 24 & $232.6 \pm 15.0$ & $193.8 \pm 15.1 * \ldots$ & $206.5 \pm 9.2^{* * *}$ \\
\hline Random plasma & 0 & $490.8 \pm 13.0$ & $500.7 \pm 14.8$ & $488.9 \pm$ II.I \\
\hline \multirow[t]{2}{*}{ glucose (mg/dL) } & 5 & $494.2 \pm 16.0$ & $454.9 \pm 20.0$ & $433.4 \pm 23.8$ \\
\hline & 24 & $364.9 \pm 30.7$ & $303.2 \pm 27.8^{* * *}$ & $317.9 \pm 29.5^{*}$ \\
\hline $\mathrm{AUC}_{\text {glucose }}$ during & 0 & $977.8 \pm 31.0$ & $952 \pm 29.1$ & $930.0 \pm 29.9$ \\
\hline \multirow[t]{2}{*}{ OGTT (mg*h/dL) } & 6 & $997.6 \pm 32.5$ & $886.5 \pm 36.8^{*, \#}$ & $813.9 \pm 68.9 *, \#$ \\
\hline & 24 & $726.1 \pm 38.7$ & $568 \pm 24.5^{* *, \#}$ & $554.9 \pm 50.6^{*, \#}$ \\
\hline Fructosamine ( $\mu \mathrm{mol} / \mathrm{L})$ & 24 & $345.5 \pm 57.0$ & $354.1 \pm 4 I .1$ & $252.3 \pm 23.2^{* * *}$ \\
\hline
\end{tabular}

Notes: Values are mean \pm SEM. $* P<0.05 ; * * P<0.0$ I; ***P $<0.1$ vs control group (Student's $t$-test); ${ }^{*} P<0.05$; ${ }^{*} P<0.1$ vs control (ANOVA followed by Dunnett's post hoc test), $\mathrm{n}=8-10$.

Abbreviations: ANOVA, analysis of variance; $\mathrm{AUC}_{\text {glucose }}$, area under the glucose response curve; OGTT, oral glucose tolerance test; SEM, standard error of mean. 
A

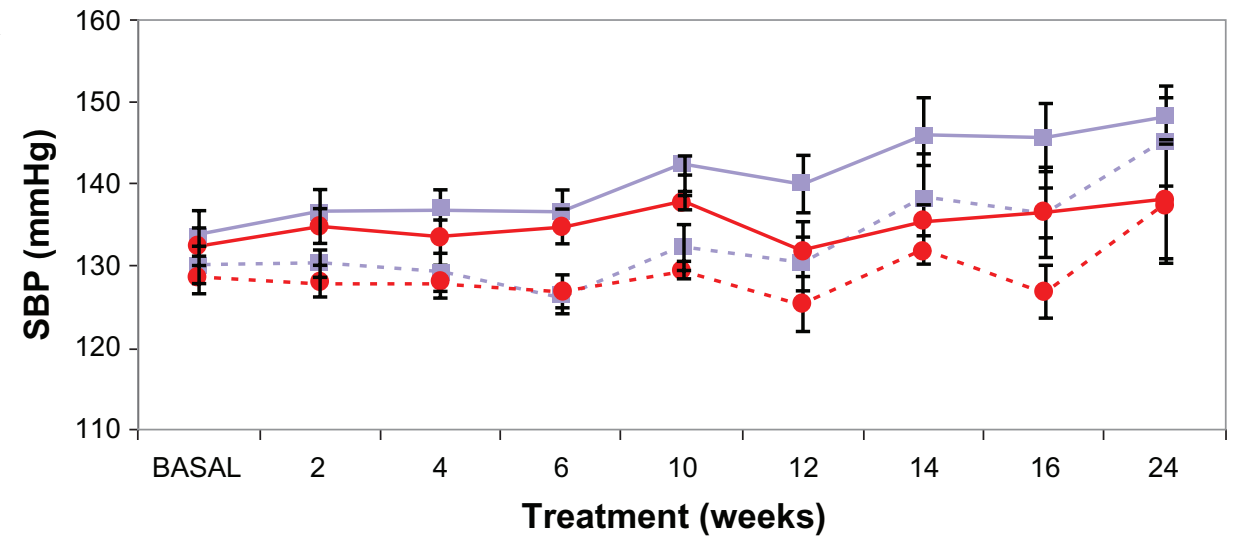

- - - - Light_VEH —-Dark_VEH

- - - - Light_TRC150094___ Dark_TRC150094

B

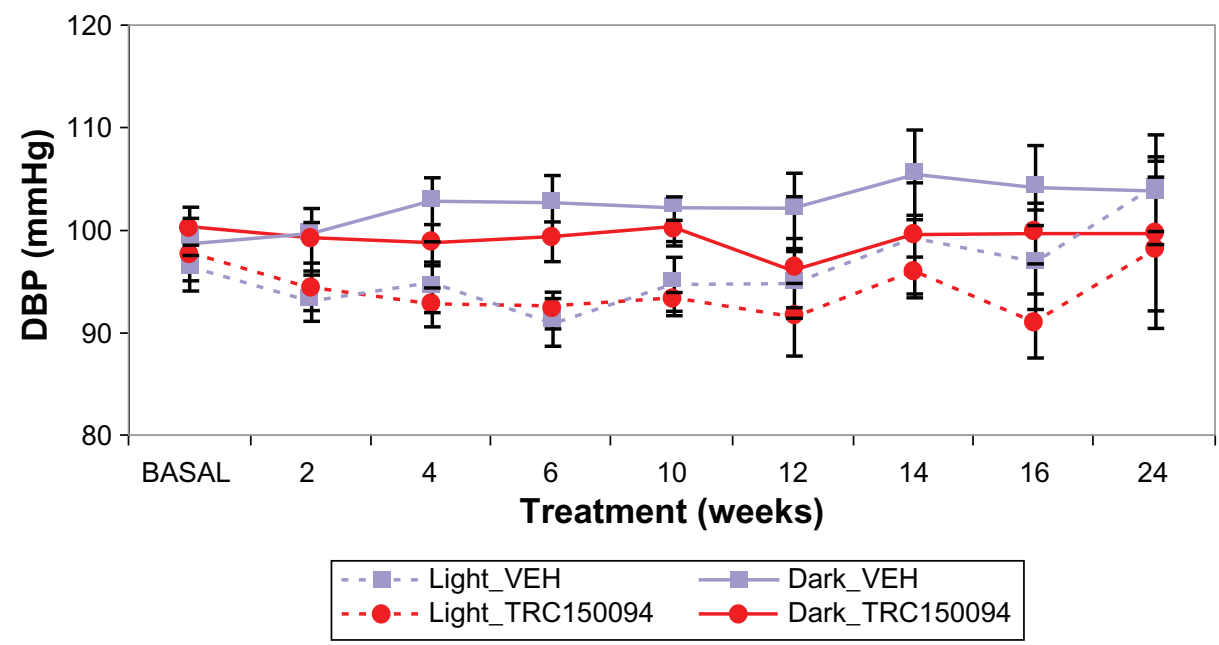

Figure 2 A) Effect of TRCI50094 (I2 mg/kg body weight) on SBP in obese ZSFI rats; B) Effect of TRCI50094 (I2 mg/kg body weight) on DBP in obese ZSFI rats. The solid line graph denotes data recorded during the night-time (dark), and the dotted line denotes data recorded during the daytime (light).

Abbreviations: DBP, diastolic blood pressure; SBP, systolic blood pressure.

TRC150094-treated group $341.2 \pm 15.9$ vs $267.9 \pm 13.6$ (pressure, gm) in the control group.

\section{Effect on mitochondrial respiration capacity to import and oxidize fatty acids in metabolically active tissues}

Respiratory parameters were measured in mitochondria isolated from the liver and skeletal muscle for both control- and TRC150094-treated obese ZSF1 rats. Significant enhancement in mitochondrial respiration (both state 3 and state 4) was observed in tissues of rats treated with TRC150094 as compared with control (Table 3).

Next, the activity of the CPT system (the rate-limiting step for mitochondrial fatty acid uptake) and the ability of mitochondria to import and oxidize fatty acids and mitochondrial FAO rate were measured in the liver and skeletal muscle of both control and TRC150094-treated obese ZSF1 rats (Table 3).

In liver and skeletal muscle, TRC150094 treatment resulted in enhancement of both CPT dependence (28\% each) and CPT independence (37\% and $46 \%$, respectively) FAO (Table 3).

\section{Discussion}

Recently, we have shown that TRC150094 has significant metabolic effects but minimal activity toward the thyroid receptors and is therefore devoid of adverse effects on the heart associated with thyroid receptor hyperactivation. The same study also demonstrates that TRC150094 increases mitochondrial FAO and respiratory chain activity and thereby increases resting metabolic rate in Wistar rats fed a highfat diet. ${ }^{16}$ In the present study, we employed male obese ZSF1 rats on a diet that was not unduly rich in fat (PURINA 5008, 


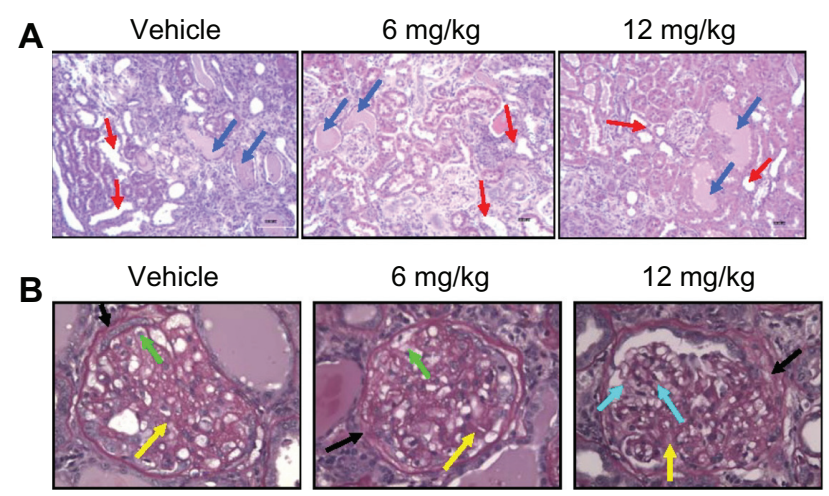

Figure 3 A) Hematoxylin and eosin-stained representative kidney sections. Control-treated obese ZSFI rat with severe nephropathy; TRCI $500946 \mathrm{mg} / \mathrm{kg}$ body weight and $12 \mathrm{mg} / \mathrm{kg}$ body weight-treated rat, respectively, with reduced grade of nephropathy. (Red arrows showing dilated tubules and blue arrows showing hyaline cast). (100× magnification) B) PAS-stained kidney sections. Glomerulus showing moderate glomerulosclerosis in control-treated and TRCI50094 $6 \mathrm{mg} / \mathrm{kg}$ body weight-treated rat, illustrated by global expansion of mesangial matrix (pink stained, yellow arrow), thickened basement membrane (black arrow) with infiltration of foam cells (green arrow). Glomerulus of $12 \mathrm{mg} / \mathrm{kg}$ showed milder glomerulosclerosis characterized by expansion of mesangial matrix (pink stained, yellow arrow) and normal capillaries and glomeruli (blue arrow) portion indicating improvement in nephropathy. $(\times 400)$ For details refer to supplementary data.

Abbreviation: PAS, periodic acid Schiff.

$7 \%$ fat) that exhibited more severe obesity, type 2 diabetes, and a cluster of other metabolic abnormalities. Male obese ZSF1 rats exhibited fairly well characterized features of metabolic syndrome such as severe obesity, hyperlipidemia (hypertryglyceridemia and hypercholesterolemia), hyperglycemia, hyperinsulinemia, and hypertension. Obese ZSF1 rats died (at the age of $\sim 12$ months) with symptoms of end-stage renal failure accompanied by marked cardiac hypertrophy. ${ }^{20}$

TRC150094 prevented the accumulation of visceral fat in obese ZSF1 rats, at least in part by increasing energy expenditure, as demonstrated in our previous study in rats fed a high-fat diet. The increase in slow oxidative-type skeletal muscle fiber enhanced mitochondrial respiratory chain activity and FAO in TRC150094-treated obese ZSF1 rats, which substantiates that a similar mechanism is responsible for an increase in energy expenditure. In the present study, we found that long-term treatment with TRC150094 not only attenuated visceral fat accumulation in obese ZSF1 rats but also positively influenced various $\mathrm{CV}$ risk components associated with obese dysglycemic patients. For instance, long-term treatment of obese ZSF1 rats with TRC150094 improved glucose tolerance and glycemic profile, attenuated a rise in blood pressure, and improved functional capacity of skeletal muscle. The obese ZSF1 rats were hyperglycemic and as age progressed they progressively developed nephropathy. The improvement in glycemic control in TRC150094-treated rats resulted in reduced glycosuria and protienurea in obese ZSF1 rats in an early treatment period (6-16 weeks of treatment period), and thus slowed down the progression of nephropathy.

In the present study, the effect on blood pressure appeared noticeable after 4-6 weeks of treatment. As there is a relationship between insulin resistance and deterioration of endothelial function, ${ }^{24}$ better blood pressure control in TRC150094-treated rats is likely mediated through improved insulin sensitivity. This finding has immense clinical relevance. A Global Cardiometabolic Risk Profile in Patients with Hypertension Disease (GOOD) survey reported that blood pressure control was significantly worse in hypertensive patients with diabetes mellitus and/or metabolic syndrome. Moreover, $95 \%$ of patients with both metabolic syndrome and type 2 diabetes had poorly controlled blood pressure. ${ }^{25}$

One additional observation from this study was that obese ZSF1 rats exhibited distinct differences in parameters such as body weight gain, fat accumulation, and lipid profile after TRC150094 treatment than a control group in

Table 3 Effect of TRCI 5009412 mg/kg/d treatment on liver and skeletal muscle mitochondrial parameters in male obese ZSFI rats

\begin{tabular}{|c|c|c|c|c|}
\hline \multirow[t]{2}{*}{ Parameter } & \multicolumn{2}{|l|}{ Liver } & \multicolumn{2}{|c|}{ Skeletal muscle } \\
\hline & Control & Treated & Control & Treated \\
\hline \multicolumn{5}{|c|}{ Respiratory parameters } \\
\hline $\begin{array}{l}\text { State } 4 \text { respiration } \\
(\mathrm{nmol} / \mathrm{mg} / \mathrm{min})\end{array}$ & $54.1 \pm 8.2$ & $68.1 \pm 6.3$ & $67.8 \pm 12.7$ & $104.7 \pm 11.35 *$ \\
\hline $\begin{array}{l}\text { State } 3 \text { respiration } \\
(\mathrm{nmol} / \mathrm{mg} / \mathrm{min})\end{array}$ & $193.5 \pm 17.5$ & $260.4 \pm 22.0 *$ & $198.4 \pm 28.8$ & $301.86 \pm 20.94 *$ \\
\hline \multicolumn{5}{|c|}{ Fatty acid oxidation (FAO) } \\
\hline $\begin{array}{l}\text { FAO/palmitoyl CoA } \\
\text { (nmol/mg/min) }\end{array}$ & $29.3 \pm 2.1$ & $37.3 \pm 3.9^{*}$ & $28.9 \pm 5.2$ & $36.96 \pm 7.66$ \\
\hline FAO/palmitoyl carnitine & $39.3 \pm 5.3$ & $53.9 \pm 3.1^{*}$ & $42.7 \pm 2.2$ & $62.4 \pm 6.3^{* *}$ \\
\hline $\begin{array}{l}\text { CPT activity } \\
\text { (nmolCoA/mg/min) }\end{array}$ & $23.98 \pm 3.0$ & $28.13 \pm 1.5$ & $17.6 \pm 2.7$ & $21.23 \pm 3.8$ \\
\hline
\end{tabular}

Notes: Values are mean \pm SEM (standard error of mean). $* P<0.05$; $* * P<0.01$ vs control group $(n \geq 5)$.

Abbreviations: CoA, coenzyme A; CPT, carnitine palmitoyl transferase; SEM, standard error of mean. 
an early treatment period ie, 6-16 weeks. However, after 16 weeks of treatment, the differences between these two groups diminished (data in Supplemental Information Tables A and B), though the mitochondrial substrate oxidation capacity (respiratory rate and FAO) remained high for the TRC150094 treatment group. This might be due to a reduction in weight gain in the control group because of a higher severity of progression of nephropathy and reduced food intake in obese ZSF1 rats.

The findings of the present study have significance in view of addressing the multiple $\mathrm{CV}$ risk factors associated with diabetes and obesity. About $85 \%$ of patients with type 2 diabetes are abdominally obese and characterized by the atherothrombotic inflammatory profile as well as a cluster of CV risks such as high LDL cholesterol, hypertension, and low HDL cholesterol. ${ }^{13}$ Due to the constellation of abnormality at multiple biochemical, metabolic, and physiological components, the progression of disease involves progressive functional abnormality of multiple vital organs. As a consequence, to aim at the optimal management of CV disease risk in type 2 diabetes, one must pay attention not only to the patient's glycemic control but also to the accompanying multiple risk factors. Despite current standards of care for individual risk factors such as LDL cholesterol, blood pressure, or glycemia, these interventions are insufficient to prevent the development or progression of microvascular disease in a significant proportion of obese type 2 diabetic patients. This has raised an urgent need for a renewed focus on developing agents that are capable of reducing the residual risk of $\mathrm{CV}$ events and microvascular complications in such patients. ${ }^{15}$ The findings of the present study showed that TRC150094 not only improved the individual component of multiple $\mathrm{CV}$ risks in the obese ZSF1 rats but also improved the associated complications, such as nephropathy and steatohepatitis.

Moreover, TRC150094 treatment also improved functional capacity of skeletal muscle. Even though physical activity, including appropriate endurance and resistance training, is a major therapeutic modality for type 2 diabetes, it has been observed that the functional exercise capacity of those with type 2 diabetes is frequently lower than agematched nondiabetic counterparts. ${ }^{26}$ In view of this, the effect of TRC150094 on skeletal muscle fibre-type composition and metabolic capacity has immense significance for type 2 diabetic subjects. Taken together, the findings of the present study reveal the potential of TRC150094 to attenuate the progression of metabolic derangement and microvascular complications associated with viscerally obese type 2 diabetes at different vital organ levels. Hence, such an integrated approach regarding the progression or development of multiple abnormalities has scope to minimize the residual $\mathrm{CV}$ risk in viscerally obese dysglycemic subjects.

\section{Conclusion}

This study demonstrates that TRC150094 attenuates the progression of hypertension, insulin resistance, dysglycemia, and atherogenic dyslipidemia, factors reported to signify significant CV risk amongst viscerally obese dysglycemic subjects. Moreover, at organ level, TRC150094 reduced steatohepatitis, reduced progression of nephropathy, and preserved cardiac contractile function.

If reproduced in humans, this pharmacological profile of TRC150094 may constitute a promising new class of molecules that may have a potential beneficial therapeutic approach for the treatment of nontraditional CV risk factors and may reduce residual risk in viscerally obese dysglycemic patients. Moreover, the observed metabolic and functional effects on skeletal muscle suggest that TRC150094 as a therapy may help to facilitate adherence to prescribed exercise, which would remain the mainstay along with diet control in such patients.

\section{Acknowledgments}

The authors are thankful to Mr Vishal Mane, Mr Kirit Waghela, Ms Binita Shah, and Ms Suchi Sharma for assisting in the experimental work and to Dr Shikha Kumar for editorial assistance. The authors are also thankful to Dr Mrugesh, Mr Midda, and Mr Amit from Gujrat Imaging Center, Ahmedabad, India, for their help with magnetic resonance imaging.

\section{Disclosure}

TRC150094 is a molecule patented under a patent owned by Torrent Pharmaceuticals Ltd. SPZ, SM, AD, RG, RCG, VC, and CD are employees of Torrent Pharmaceuticals Ltd. The authors report no other conflicts of interest in this work.

\section{References}

1. Savage DB, Petersen KF, Shulman GI. Disordered lipid metabolism and the pathogenesis of insulin resistance. Physiol Rev. 2007;87(2): 507-520.

2. Bianchi C, Penno G, Pancani F, et al. Non-traditional cardiovascular risk factors contribute to peripheral arterial disease in patients with type 2 diabetes. Diabetes Res Clin Prac. 2007;78(2):246-253.

3. Despres JP, Lemieux I, Bergeron J, et al. Abdominal obesity and the metabolic syndrome: contribution to global cardiometabolic risk. Arterioscler Thromb Vasc Biol. 2008;28(6):1039-1049.

4. Bugianesi E, Zannoni C, Vanni E, Marzocchi R, Marchesini G. Nonalcoholic fatty liver and insulin resistance: a cause-effect relationship? Dig Liver Dis. 2004;36(3):165-173. 
5. Duvnjak M, Lerotic I, Barsic N, Tomasic V, Virovic Jukic L, Velagic V. Pathogenesis and management issues for non-alcoholic fatty liver disease. World J Gastroenterol. 2007;13(4):4539-4550.

6. Enas AE, Mohan V, Mohan D, Syed F, Pazhoor S, Chennikkara H. The metabolic syndrome and dyslipidemia among Asian Indians: a population with high rates of diabetes and premature coronary artery disease. J Cardiometab Syndr. 2007;2(4):267-275.

7. Aristizabal D, Gallo J, Fernandez R, Restrepo MA, Zapata N, Correa M. The insulin gradient phenomenon: a manifestation of the effects of body weight on blood pressure and insulin resistance. J Cardiometab Syndr. 2008;3(4):218-223.

8. Fujita T. Insulin resistance and salt-sensitive hypertension in metabolic syndrome. Nephrol Dial Transplant. 2007;22(11):3102-3107.

9. Franco OH, Massaro JM, Civil J, Cobain MR, O’Malley B, D'Agostino RB Sr. Trajectories of entering the metabolic syndrome: the framingham heart study. Circulation. 2009;120(20): 1943-1950.

10. Hillier TA, Rousseau A, Lange C, et al. Practical way to assess metabolic syndrome using a continuous score obtained from principal components analysis: the D.E.S.I.R. Cohort. Diabetologia. 2006;49: 1528-1535.

11. Despres JP. Targeting abdominal obesity and the metabolic syndrome to manage cardiovascular disease risk. Heart. 2009;95(3): 1118-1124.

12. Arbeeny CM. Addressing the unmet medical need for safe and effective weight loss therapies. Obes Res. 2004;12(8):1191-1196.

13. LaRosa JC, Grundy SM, Waters DD, et al. Intensive lipid lowering with atorvastatin in patients with stable coronary disease. $N$ Engl J Med. 2005;352(14):1425-1435.

14. Ferreira SR. Revisiting clinical trials on glycemic control and cardiovascular risk. Diabetol Metab Syndr. 2009;1(1):12.

15. Fruchart JC, Sacks FM, Hermans MP, et al. The Residual Risk Reduction Initiative: a call to action to reduce residual vascular risk in dyslipidaemic patients. Diab Vasc Dis Res. 2008;5(4):319-325.
16. Cioffi F, Zambad SP, Chhipa L, et al. TRC150094, a novel functional analogue of iodothyronines, reduces adiposity by increasing energy expenditure and fatty acid oxidation in rats receiving a high-fat diet. FASEB J. 2010;24(9):3451-3461.

17. Dominguez J, Wu P, Packer CS, Temm C, Kelly KJ. Lipotoxic and inflammatory phenotypes in rats with uncontrolled metabolic syndrome and nephropathy. Am J Physiol Renal Physiol. 2007;293(3): F670-F679.

18. NIH. Guide for the care and use of laboratory animals. NIH Publication No. 85-23. Revised 1985.

19. CPCSEA. CPCSEA guidelines for laboratory animal facility: committee for the purpose of control and supervision on experiments on animals. Ind J Pharmacol. 2003;35:257-274.

20. Tofovic SP, Kusaka H, Kost CK Jr, Bastacky S. Renal function and structure in diabetic, hypertensive, obese ZDFxSHHF-hybrid rats. Ren Fail. 2000;22(4):387-406.

21. Lanni A, Moreno M, Cioffi M, Goglia F. Effect of 3,3'-diiodothyronine and 3,5-diiodothyronine on rat liver oxidative capacity. Mol Cell Endocrinol. 1992;86(3):143-148.

22. Luna LG. Manual of Histologic Staining Methods of the Armed Forces Institute of Pathology. 3rd ed. New York: McGraw-Hill Book Company; 1968.

23. Joshi D, Gupta R, Dubey A, et al. TRC4186, a novel AGE-breaker, improves diabetic cardiomyopathy and nephropathy in Ob-ZSF1 model of type 2 diabetes. J Cardiovasc Pharmacol. 2009;54(1):72-81.

24. Nisoli E, Clementi E, Carruba MO, Moncada S. Defective mitochondrial biogenesis: a hallmark of the high cardiovascular risk in the metabolic syndrome? Circ Res. 2007;100(6):795-806.

25. Zidek W, Naditch-Brule L, Perlini S, Farsang C, Kjeldsen SE. Blood pressure control and components of the metabolic syndrome: the GOOD survey. Cardiovasc Diabetol. 2009;8:51.

26. Regensteiner JG, Bauer TA, Reusch JE, et al. Abnormal oxygen uptake kinetic responses in women with type II diabetes mellitus. $J$ Appl Physiol. 1998;85(1):310-317. 


\section{Supplementary information}

\section{Body weight, food intake, and plasma lipid profile data Histopathological and histochemical evaluation of kidney}

Tissue preparation: neutral-buffered formalin-fixed kidney tissues were trimmed at $2-3 \mathrm{~mm}$ thickness, processed in graded isopropyl alcohol, cleared in xylene, and infiltrated with paraffin. Paraffin-embedded 4-micron-thick serial kidney sections were taken and stained with either hematoxylin and eosin, periodic acid Schiff (PAS), or Masson's trichrome staining. Glomerulosclerosis was evaluated in a PAS-stained kidney section; nephropathy and tubule-interstitum changes were evaluated in hematoxylin and eosin-stained and Masson's trichrome-stained kidney sections under light microscopy on a grade scale of 0 to 4 by a pathologist who was blinded to the treatment groups.

\section{For glomerulosclerosis index (GSI)}

In a PAS-stained kidney section, 100 glomeruli from each animal were evaluated on the scale of grade $(G) 0$ to 4 for glomerulosclerosis. G0 normal glomeruli without any change; G1 minimal glomerulosclerosis characterized by the focal expansion of mesangial matrix and thickening of basement membrane; G2 mild glomerulosclerosis characterized by the global expansion of mesangial matrix and thickening of basement membrane with an increase in cellularity; G3 moderate glomerulosclerosis characterized by the global expansion of mesangial matrix and thickening of basement membrane with an increase in cellularity, infiltration of foam cells, and hypertrophied podocytes and epithelial cells; G4 severe glomerulosclerosis characterized by a more severe form of G3 glomerulosclerosis or completely sclerosed or obsolescence of glomeruli. An index of glomerulosclerosis for each animal has been derived by:

$\mathrm{GSI}=(\mathrm{G} 0 \times 0)+(\mathrm{G} 1 \times 1)+(\mathrm{G} 2 \times 2)+(\mathrm{G} 3 \times 3)+(\mathrm{G} 4 \times 4)$

Total number of glomeruli scored.

Where $\mathrm{G} 0, \mathrm{G} 1, \mathrm{G} 2, \mathrm{G} 3$, and $\mathrm{G} 4$ are the total no of glomeruli showing respective grade glomerulosclerosis.

\section{Measurement of CV functional parameters}

Measurement of cardiac function was performed terminally in the obese ZSF1 rats implanted with telemetry transmitter using Millar Pressure Volume (PV) System (Millar Instruments, Houston, TX, USA). ${ }^{20}$ These data were generated to assess whether chronic treatment in the disease model influences cardiovascular function adversely. A microtip PV catheter (SPR-838) was inserted into the right carotid artery and advanced into the left ventricle (LV). A polyethylene catheter was inserted into the left jugular for fluid administration. After stabilization for 10-20 minutes, LV-PV signals were recorded continuously at a sampling rate of 1000 per second using an MPVS-300 conductance system (Millar Instruments) coupled to a PowerLab 8/30 (ADInstruments, NSW, Australia). $50 \mu 1$ of $20 \%$ saline was injected intravenously so as to establish a parallel conductance volume from a shift of PV relations, and this was used for correction for the cardiac mass volume. LV-PV relations were also captured by transiently compressing the inferior vena cava.

LV parameters were computed using cardiac $\mathrm{PV}$ analysis software (PVAN3.2, Millar Instruments). Volume calibrations were performed with a Millar volume calibration cuvette, which consists of a 1-cm-deep cylindrical block with cylindrical holes of known diameters ranging from $2 \mathrm{~mm}$ to $11 \mathrm{~mm}$ filled with fresh heparinized whole rat blood. The linear volume-conductance regression of the absolute volume in each cylinder versus the raw signal acquired by the conductance catheter was used for the volume calibration.

The results indicated that the cardiac functional parameters recorded showed no deterioration with TRC150094 treatment in comparison with the control group. Instead, a trend towards better preserved cardiovascular function was observed in the TRC150094-treated group in comparison with the control group (Table E). 
Table A Effect of TRCI50094 on body weight gain and food intake in male obese ZSFI rats

\begin{tabular}{|c|c|c|c|c|}
\hline Parameter & Treatment week & Control & $6 \mathrm{mg} / \mathrm{kg}$ & $12 \mathrm{mg} / \mathrm{kg}$ \\
\hline \multirow[t]{3}{*}{ Body weight gain (g) } & 6 & $110.1 \pm 6.21$ & $94.9 \pm 3.7^{*}$ & $97.1 \pm 5.6 * * *$ \\
\hline & 16 & $177.9 \pm 11.0$ & $170.7 \pm 5.8$ & $155.2 \pm 5.6^{*}$ \\
\hline & 24 & $207.1 \pm 13.9$ & $203.2 \pm 6.6$ & $199.6 \pm 10.6$ \\
\hline \multirow[t]{4}{*}{ Food intake (g/day) } & 0 & $40.5 \pm 3.1$ & $42.7 \pm 1.0$ & $41.3 \pm 1.6$ \\
\hline & 6 & $39.6 \pm 1.6$ & $39.4 \pm 0.9$ & $38.4 \pm 1.4$ \\
\hline & 16 & $35.2 \pm 2.2$ & $35.7 \pm 1.0$ & $33.9 \pm 2.0$ \\
\hline & 24 & $30.6 \pm 1.6$ & $30.9 \pm 1.2$ & $31.7 \pm 1.9$ \\
\hline
\end{tabular}

Notes: Values are mean \pm SEM (standard error of mean). $* P<0.05$; *** $P<0.1$ vs control group.

Table B Effect of TRCI50094 on plasma lipid parameters in male obese ZSFI rats

\begin{tabular}{|c|c|c|c|c|}
\hline \multirow[t]{2}{*}{ Parameter } & \multirow[t]{2}{*}{ Treatment week } & \multicolumn{3}{|l|}{ Male } \\
\hline & & Control & $6 \mathrm{mg} / \mathrm{kg}$ & $12 \mathrm{mg} / \mathrm{kg}$ \\
\hline \multirow[t]{4}{*}{ Fasting plasma TG } & 0 & $2547 \pm 213$ & $2497 \pm 193$ & $2596 \pm 142$ \\
\hline & 6 & $3471 \pm 419$ & $3|2| \pm 306$ & $3035 \pm 168$ \\
\hline & 16 & $6327 \pm 750$ & $4974 \pm 778$ & $5499 \pm 555$ \\
\hline & 24 & $8278 \pm|33|$ & $8056 \pm 949$ & $8147 \pm 1590$ \\
\hline \multirow[t]{4}{*}{ Fasting plasma TC } & 0 & $233.2 \pm 8.8$ & $220.8 \pm 7.3$ & $231.1 \pm 8.2$ \\
\hline & 6 & $349.2 \pm 21.2$ & $306.6 \pm 18.8$ & $289.5 \pm 17.5^{*}$ \\
\hline & 16 & $632.1 \pm 58.0$ & $612.4 \pm 78.9$ & $585.4 \pm 54.3$ \\
\hline & 24 & $704.5 \pm 91.0$ & $820.8 \pm 85.5$ & $760.7 \pm 106.5$ \\
\hline \multirow[t]{4}{*}{ NEFA } & 0 & $0.85 \pm 0.08$ & $0.97 \pm 0.10$ & $0.98 \pm 0.10$ \\
\hline & 6 & $1.01 \pm 0.05$ & $0.86 \pm 0.08$ & $1.01 \pm 0.12$ \\
\hline & 16 & $0.91 \pm 0.05$ & $0.86 \pm 0.04$ & $0.75 \pm 0.05^{*}$ \\
\hline & 24 & $3.7 \mathrm{I} \pm 0.32$ & $3.22 \pm 0.45$ & $3.27 \pm 0.33$ \\
\hline
\end{tabular}

Notes: Values are mean \pm SEM. $* P<0.05$ vs control group.

Abbreviations: NEFA, non-essential fatty acid; SEM, standard error of mean; TC, total cholesterol; TG, triglycerides.

Table C Effect of TRCI50094 on urinary microalbumin to creatinine ratio in male obese ZSFI rats

\begin{tabular}{|c|c|c|c|c|}
\hline \multirow[t]{2}{*}{ Treatment } & \multicolumn{4}{|c|}{ Treatment week } \\
\hline & 0 & 6 & 16 & 24 \\
\hline Control & $16.9 \pm 2.9$ & $246.0 \pm 19.4$ & $356.7 \pm 24.4$ & $454.6 \pm 45.1$ \\
\hline TRCI50094, 6 mg/kg & $15.6 \pm 2.2$ & $210.5 \pm 18.2$ & $329.8 \pm 23.3$ & $460.2 \pm 45.1$ \\
\hline TRCI50094, 12 mg/kg & $11.4 \pm 2.3$ & $190.2 \pm 11.6 *$ & $287.2 \pm 23.0 \mathrm{I} *$ & $280.5 \pm 49.4^{*}$ \\
\hline
\end{tabular}

Notes: Values are mean \pm SEM (standard error of mean). $* P<0.05$ vs control group.

Table D Glomerulosclerosis Index (GSI) and mean severity of nephropathy

\begin{tabular}{|c|c|c|c|c|c|}
\hline \multicolumn{3}{|l|}{ GSI } & \multicolumn{3}{|c|}{ Nephropathy } \\
\hline Control & TRCI 50094 & TRCI50094 & Control & TRCI50094 & TRCI50094 \\
\hline & $6 \mathrm{mg} / \mathrm{kg}$ & $12 \mathrm{mg} / \mathrm{kg}$ & & $6 \mathrm{mg} / \mathrm{kg}$ & $12 \mathrm{mg} / \mathrm{kg}$ \\
\hline $2.01 \pm 0.07$ & $2.07 \pm 0.08$ & $1.83 \pm 0.06$ & $2.39 \pm 0.29$ & $2.33 \pm 0.17$ & $1.92 \pm 0.23$ \\
\hline
\end{tabular}

Note: Values are mean \pm SEM (standard error of mean). 
Table E Effect of TRCI50094 on cardiovascular functional parameters in male obese ZSFI rats

\begin{tabular}{|c|c|c|}
\hline Parameters & Control & $\begin{array}{l}\text { TRCI50094 } \\
\text { I } 2 \text { mg/kg }\end{array}$ \\
\hline Heart rate (beats/minute) & $288.6 \pm 12.0$ & $288.0 \pm 22.5$ \\
\hline Cardiac output ( $\mu \mathrm{L} /$ minute) & $15|3| .7 \pm 774.7$ & $16895.5 \pm 2371.2$ \\
\hline$+\mathrm{dP} / \mathrm{dt}(\mathrm{mmHg} / \mathrm{second})$ & $7576.4 \pm 1286.6$ & $8432.5 \pm 571.2$ \\
\hline$-\mathrm{dP} / \mathrm{dt}(\mathrm{mmHg} / \mathrm{second})$ & $7024.6 \pm 971.1$ & $8198.3 \pm 1025.4$ \\
\hline LV pressure decay, Tau (millisecond) & $12.3 \pm 0.7$ & $12.1 \pm 0.9$ \\
\hline ESPVR slope $(\mathrm{mmH} / \mu \mathrm{L})$ & $1.3 \pm 0.2$ & $1.8 \pm 0.4$ \\
\hline EDPVR slope $(\mathrm{mmHg} / \mu \mathrm{L})$ & $0.07 \pm 0.01$ & $0.06 \pm 0.003$ \\
\hline PRSW (mmHg) & $66.9 \pm 11.9$ & $102.9 \pm 25.9$ \\
\hline
\end{tabular}

Notes: Values are mean \pm SEM (standard error of mean). $n=4-5$.

Abbreviations: EDPVR, end diastolic pressure-volume relationship; ESPVR, end systolic pressure-volume relationship; LV, left ventricular; PRSW, pre-recruitable stroke work.

\section{Publish your work in this journal}

Diabetes, Metabolic Syndrome and Obesity: Targets and Therapy is an international, peer-reviewed open-access journal committed to the rapid publication of the latest laboratory and clinical findings in the fields of diabetes, metabolic syndrome and obesity research. Original research, review, case reports, hypothesis formation, expert opinion and commentaries are all considered for publication. The manuscript management system is completely online and includes a very quick and fair peer-review system, which is all easy to use. Visit http://www.dovepress.com/testimonials.php to read real quotes from published authors.

Submit your manuscript here: http://www.dovepress.com/diabetes-metabolic-syndrome-and-obesity-targets-and-therapy-journal 